\title{
Spiral model of procedural cycle of educational process management
}

\author{
Valery I. Bezrukov ${ }^{1, *}$ and Elena V. Lukashina ${ }^{2}$ \\ ${ }^{1}$ MCPU, Samara, Russia, \\ ${ }^{2}$ MSU, the Faculty of Global Studies, Moscow, Russia
}

\begin{abstract}
The article analyzes the nature and characteristics of the spiral model Procedure educational systems management cycle. The authors identify patterns between the development of information and communication technologies and the transformation of the education management process, give the characteristics of the concept of "information literacy" and "Media Education". Consider the design function, determine its potential in changing the traditional educational paradigm to the new - information.
\end{abstract}

In modern conditions information culture and education represent the leading determinant of professional and everyday activities of people. It also characterizes the ability to recognize and develop the modern picture of the world. People have to adapt to the new information space. Here we see the evidence of a mismatch between the increased requests for information of individuals and their degree of satisfaction. Optimization of human communication with a source of information allows resolving this contradiction. It is enough to increase the effectiveness of interaction between them in the presence of the scientific theory of information culture.

Information Culture requires from a modern young man new knowledge and skills, a particular style of thinking, providing the necessary adaptation to social change and ensuring a worthy place in the information environment. Today, the formation of information culture of the society takes place as an unmanaged (spontaneous) process which is necessary to be structured and organized. This will allow it to become an instrument of development and adaptation to the new information environment, a way to harmonize the inner world in the development of the total volume of socially significant information [1].

Significant changes took place in the national education system over the past decade and a half. Which of the innovation do student youth give a clear preference today? The answer is obvious: the modern information and communication technologies. For the new generation mobile devices, tablets, a variety of cloud computing, ebooks and apps became habitual means of a steady access to the lifelong learning, while the training material with high-quality video, sound and graphics - natural attributes of getting knowledge.

The commitment of the younger generation for information-rich educational environment may be stipulated by other reasons. Practice shows, software, construction of which is a complex process of modeling, design, modularity and systemacy, leads the student to find a productive, fundamentally new solutions of learning task, and on the contrary, the formal transfer of traditional textbooks to electronic media demonstrates a negative educational effects.

Expanding the range of influence, and the growing impact of the mass media (press, TV, radio, cinema, video, etc.) for the citizens' lives led to a corresponding reaction of the education system. This has resulted in the formation of a special trend in pedagogy - media education. According to the Russian Pedagogical Encyclopedia, media education is focused on the study of rules of mass communication (press, television, radio, cinema, video, etc...).

The main tasks of media education is to prepare the next generation for life in the modern information environment, the perception of a variety of information, to teach a man to understand it, to be aware of the consequences of its impact on the psyche, to master the methods of communication on the basis of non-verbal forms of communication with the help of technical means [2]. The need for media education has increased with the development of the so-called "new media" or electronic (web) media: the Internet, Internet TV, Internet radio [3].

The concept of "information literacy" is widely used now, it having a comprehensive nature. It includes the skills in handling of a range of modern information devices, as well as the functioning of the information tools. In today's world, the need to find a particular literature in printed form becomes obsolete. Information technologies come to a new level of information, where the education process occurs without any cost.

The term "information literacy" is widely spread internationally, but its use in domestic practice is associated with the limitations that have psychological and linguistic nature. The fact is that in the Russian

Corresponding author: bzrkv@hotmail.com 
language the word "literacy", meaning the ability to read and write, is associated only with the most simple, the initial level of education. Thus, the complex phenomenon of interaction of man and information is seen elementary and primitive.

In addition, the content of the concept of information literacy does not explicitly focus on a person's identity, providing a clear understanding of why he needs the information knowledge and skills and how they can and should be disposed. Thus, the limitation of the term "information literacy", on the one hand, and the ambiguity of the interpretation of the concept "information culture", the lack of clearly defined principles, goals and objectives, means and conditions of formation of information culture, on the other hand, predetermine the necessity of the development of a coherent conceptual platform in order to further the development of information education system [4].

The formation of the information environment requires forming the path of development of education corresponding the dynamic changes taking place in nature and society in terms of growing information. Innovative paradigm of modern system of education changes the paradigm of "supporting" or "enlightening" education. It becomes the most important component of the idea of "education throughout life" or lifelong learning.

The implementation of this idea is focused on overcoming the basic contradiction between the rapid pace of knowledge growth in the modern information world, its universal accessibility and at the same time, the limited abilities of a person in their assimilation of the period of study. This contradiction makes the educational institutions, first of all, form the ability to learn, to obtain information, to extract from it the necessary knowledge. To do this, the teacher must not only possess special knowledge of information, but also to be trained to pass them on. Thus, the students' information culture is being formed. Only under this condition the idea of lifelong learning can be realized [4].

The increasing amount of non-prototypical class of tasks in the life of education system determines the intensification of the process tasks (tension) of the traditional functions of management. The formal nature of the administrative activity, expressing itself in an effort to systematize and standardize the process of resolving the problems, gives way to the function of designing, the essence of which is reflected in the logic of thoughtactivity of building axiological, content-related, procedural formations and their practical implementation across the whole structure of management functions.

Education Management has long been evolving in the mode of extrapolation of habitual trends that are suitable for stable functioning, pre-defined path of development. Any manifestation of instability is governed, as a rule, by force of administrative influence. In traditional management paradigm an attitude is visibly detected in relation to the design as a secondary function for controlling the process of improving the education system. As a result, in the conditions of qualitative transformations, design as a kind of administrative activity suffers from structural and technological uncertainty, the consequence of which is the randomness in the choice of control actions, means and methods, the lack of targeted communication between them, the local nature of managerial processes of the development processes.

Today, the potential of the spontaneous development of the control system are largely exhausted. Orientation on functional specialization, strict regulation of management processes, not their results, exaggerated use of standard solutions becomes inefficient. Focus on polysubject nature of management for the activities processes become more systematic. Creation of information and telecommunications networks ensure full participation of a wide range of specialists in the management. Habitual way of professional activity of the administrator changes transforming the stereotypes of his behavior.

The specification of targets for alternating pedagogical systems, requiring a significant amount of additional resources, updates the design as a management tool, which makes teaching community concentrates its efforts on the priority issues of education development. Overcoming stochastic processes regularly occurring in the holistic variety of education system leads to the need for a clear vision of the future state of the managed object [5]. The design is intended to specify the options for management activities in order to guide the implementation directing actions, and determining the dynamics of changes for state of education system. The new educational paradigms are the least susceptible to stereotypic management decisions. In this regard, the design is claimed as an activity productive in the development and application of innovative, nonprototypical management approaches.

The function of management is the totality of relatively independent, interdependent and consistently implemented management activities. Stable connections and relationships between them form the structure of the management cycle, in which changes take place and conditions for the transfer of the object into a qualitatively new state appear. The structure of any system is the most conservative element. However, this conservatism is explained by objective requirements for the preservation of stability of the system.

However, maintaining the static state, the control loop structure can't remain unchanged. We prove this statement based on the hypothetical idea of the possible ways to update the functional management structure. Analysis of the grounds of classification models of management process proposed by Y.A. Konarzhevski, V.S. Lazarev, M.M. Potashnik, N.F. Talyzina, P.I. Tretyakov, B.C. Shakurov, T.I. Shamova, V.A. Yakunin and other scientists, has allowed us to identify the following:

1. The functions of management teaching systems as components of the structural components of a single cycle are sophisticated independent formations, the content and the structure of which is constantly being improved and developed. This process is particularly noticeable during the innovative changes. The powerful influence of external factors initiates an active 
construction and use of new methods, tools, effects in management, contributes to the specification of individual functions of the components, and its integrity. Over the last decade specific kinds of administrative activity were singled out in the theory of management: human resources management, quality management, project management, etc.

2. The diversity of quantitative classification for the grounds of functional management structure increases. This process is due, on the one hand, to the expansion of the approaches that accent the aspects of a complex, controversial and multi-level nature of the modern management activity; on the other hand, to the approaches focused on patterns and needs of a multiparadigmatic character of the object of management. Classification of foundation which organically combines both directions, are of a paramount importance for the theory and practice of management.

3. There are models of the hierarchical representation of the structure for functional composition of management, due to the desire of experts to overcome the narrowness of operationally-phased approach. The control functions are differentiated by levels in this variant of the construction of the classification foundations. Target (strategic) functions are distinguished at the highest level and the operational (tactical) ones at the lowest level. Operating function not having an independent value, obey directly the objective function. As a result of the control process, it is difficult to form hierarchically interrelated functions.

Describing the upgrade of structural options for the functional management formation, it should be noted that, offering innovative classification grounds, experts eventually fix the change order and composition of functions, but do not examine the dynamics of change in the very structure of the management cycle.

Application of the method of immanent upgrade has allowed us to establish that, provided the introduction of the design of the control the linear principle of the administrative activity gives way to objectively existing spiral cycle of the development for management process. Applying to the function of designing at the initial and subsequent turn of the management cycle is due to the necessity of a clear vision of all components of the management process, particularly at the solution of nonprototypical class of problems. The optimal version for the solutions of arising problems is created based on the function of designing [5].

Permanent collecting and analyzing of additional information identifies compliance/non-compliance for the goal set, predicts the final results of operations, specifies the source data, adjusts plans and programs, models of organizational management structure includes stakeholders - the subjects of management - into the execution of the works, which ultimately leads to a reasoned choice for the problem solution.

The study of improving, conversion and transformation observed at present in the management of teaching systems, allowed us to determine that the design function, conjugating with the boundaries of these processes, differentiates in a variety of its manifestations, depending on the degree of satisfaction of the subject with the state of the controlled system.

Subject to a satisfactory state of qualitative and quantitative parameters of the system design is implicit in each functional stage of the management of pedagogical systems. The draft plan, program, analysis, etc. is a plan for the implementation of which the subject of management needs to take both organizational and managerial solutions to ensure the process of improving the control system.

Provided that the qualitative but not the quantitative parameters of the system satisfy the subject of management, the draft of its future state is worked out retaining the basic qualitative and transformation (within the measures limits) of quantitative characteristics. In this case, the design provides modernization of the controlled object. The results are predictable and standardized, since typological identity of the system is preserved.

Given the poor state of the managed subsystem model with new qualitative parameters is developed. The design is activated in the status of a leading management function, given that the system is unable to cope with the solution of new problems, despite the presence of a high degree of functional elements of the system and its adequacy in compensatory functions. Targeted effects on quantitative parameters of the elements of the system lead to their qualitative transformation, and hence, to the subsequent transformation of its properties and relations of the controlled subsystem [5].

From the perspective of a systemic approach consistent change in the properties, relations and functions apply to all of the functionality of design possibilities. The basis for this statement is the adoption of pedagogical reality as a complex of heterogeneous, multi-level, but interrelated and interacting modules [5]. Due to this, in many aspects formal approach, it became possible to consider various aspects of the design for the managing and managed sub-systems from the unified point of view.

\section{References}

1. G.V. Polyakova, Information culture in modern society: philosophical aspects. (Sciences, Moscow, 2014) [In Rus]

2. Russian Pedagogical Encyclopedia: in 2 volumes (Great Russian Encyclopedia, Moscow, 1993) [In Rus]

3. N.I. Gendina, Pedagogy, Psychology, 1 (2012)

4. N.I. Gendina, Open Education, 1 (2007)

5. V.I. Bezrukov, Design management teaching systems: methodology, theory, practice (Moscow, 2005) [In Rus] 